\title{
Balloons, Boats and Ponies: Interface manipulation style and learning in a constraint- based planning task
}

\author{
Shirley J. Holst, Elizabeth F. Churchill and David J. Gilmore \\ Psychology Dept \\ University of Nottingham \\ Nottingham, NG7 2RD, UK \\ sjh@psychology.nottingham.ac.uk; churchill@pal.xerox.com; \\ dg@psychology.nottingham.ac.uk
}

\begin{abstract}
The role of the interface in structuring student learning and in directing the learner's cognitive effort toward relevant material has become increasingly centralised in recent years. Specifically, interface designers have been called on to design interfaces which keep the learner focused on or "engaged" in the domain material, with the view that such engagement will have learning benefits. In this paper we operationalise the notion of cognitive engagement in terms of familiar cognitive psychological principles: planning and learning. Following laboratory studies, we have taken the view that cognitive planning can be induced by increasing the "cost" of selecting and executing actions by designing less direct interfaces. A study is reported which contrasts direct and indirect manipulation interfaces for a constraint-based planning task. Results from the study suggest that manipulation style can indeed affect users' information search and task solution strategies.
\end{abstract}

KEYWORDS: empirical, education, input devices, interaction design, theory, 'cognitive cost'

\section{INTRODUCTION}

Case studies of educational software indicate that children can successfully complete computer based tasks, whilst retaining little domain knowledge (e.g. maths; Moore, 1993). For cognitive psychologists, educators and educational software developers this is clearly a worry, as more educational software appears in schools. To overcome such problems, design guidelines encourage developers to ensure that the learner is active

Human-Computer Interaction: INTERACT'97 S. Howard, J. Hammond \& G. Lindgaard (editors)

Published by Chapman \& Hall OIFIP 1997 
schools. To overcome such problems, design guidelines encourage developers to ensure that the learner is active (Jackson et al, 1996), engaged (Norman and Sporher, 1996), "cognitively immersed" in the material or motivated (Mallone, 1981). In this paper we report an attempt to draw on sound psychological theory and practice to operationalise the notion of cognitive engagement in terms of familiar cognitive psychological principles: planning and learning. Here, the role of the interface in structuring students' cognitive activities is centralised. Far from being a seen as a passive window onto the domain (as suggested for example by Laurillard, 1993), the interface and interaction style are seen as important for structuring learning activities; students' attention and cognitive processes can be directed to appropriate material for reflection through careful design of the interface and interaction style. In the following sections, we elaborate on this and present a comparative study.

\subsection{Interfaces and learning}

Studies indicate that increasing the 'cost' of actions by providing indirect, command-based interfaces and by increasing delays in system response encourages active problem solving and facilitates retention, recall and transfer of information. Svendsen (1991) and O'Hara and Payne (1994) empirically supported their prediction that certain forms of cost improve learning on computerbased problem-solving tasks. Command-based interfaces, delayed feedback and difficult error-recovery routines actually enhanced learning. Conversely, increasing ease-of-use through direct manipulation results in recognition or display-based performance (Svendsen, 1991; O'Hara and Payne, 1994; Gilmore, 1996). Such counter-intuitive results can help to explain why case studies like that cited above demonstrate little transfer of domain material away from the interface.

Applying this to the development of educational software, this analysis suggests that learning can be best facilitated by making the interface less easy-to-use for certain tasks by reducing the directness of the interface manipulation and thus forcing explicit cognitive processes like planning and problem solving (Churchill and Ainsworth, 1995; Gilmore, 1996). In this view, it is suggested that way in which the learner physically manipulates objects on-screen will affect the focus of their cognitive activity. By 'forcing' the learner to cognitively process curriculum appropriate information, concept formation and domain knowledge retention will be facilitated. There is some evidence from evaluation of intelligent tutoring systems that this assertion is wellfounded; students using menus performed worse on post tests than students who were required to type responses into the tutor (Corbett, Anderson and Fincham, 1990).

Although these results are promising, there is still a need to understand fully the extent of this effect, to develop a clearer understanding of this effect and to consider the extent to which it "scales up" to more complex problem solving domains. In this vein, we studied a constraint based planning task to observe whether interface design and manipulation style affects students' information seeking behaviours, pre-action planning and post task knowledge.

\section{HONEY BEARS TASK}

\subsection{Introduction}

From the above discussion, we predicted that a less direct interface manipulation style will result in more "cognitive cost" in the form of pre-planning before action. To empirically test this hypothesis, two interfaces were developed for an on-line, constraint based planning task couched in the form of an adventure game: the Honey Bears task (Littleton et al, 1994).

\section{The task and interfaces}

In the Honey Bears task three bears (Airbear, Waterbear and Ponybear) plan a picnic, but on arrival at their destination (Almwood) discover they have forgotten their honey. The honey is at Flint on the other side of the river, but in the river there are Honey Monsters who will steal the honey if the bears attempt to move it by boat (see Figure 1). The goal of the task is for bears to use appropriate means of transport (boat, balloon or pony) to get the honey, whilst avoiding the Honey Monsters. Clearly the task requires planning of a route, whilst ensuring that the "correct" bear is in the appropriate place to use the most appropriate form of transport. (e.g. Ponybear can only use the pony and so on) 


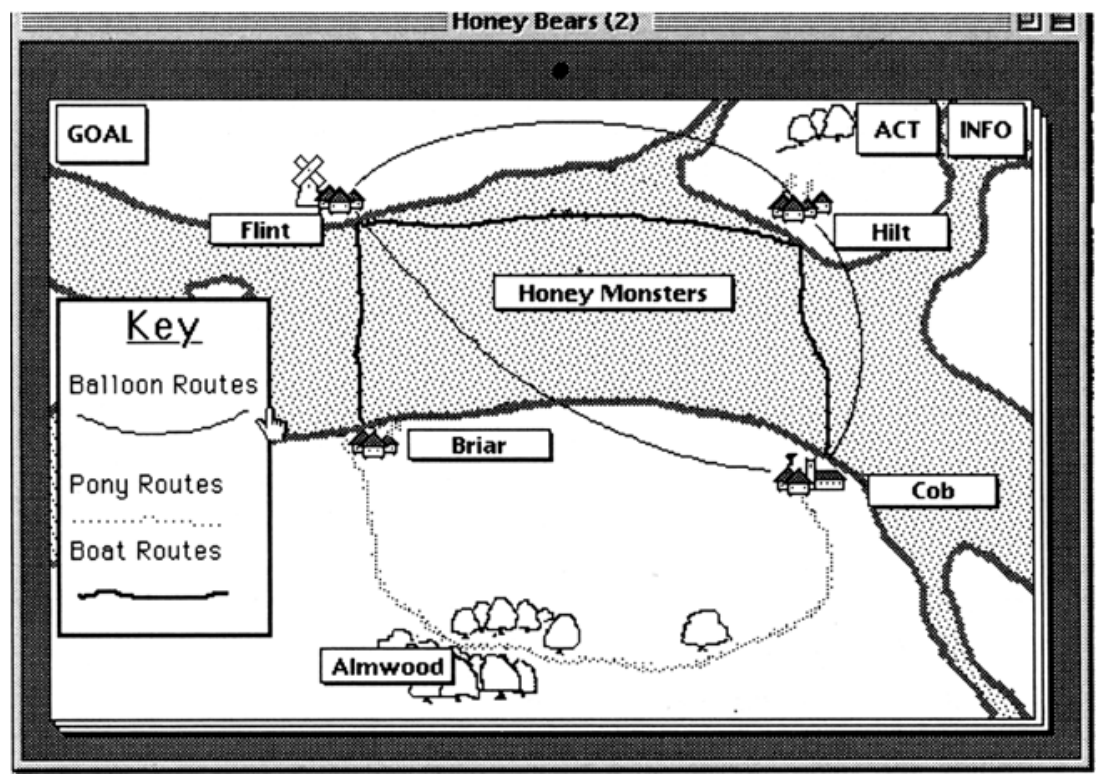

Figure 1: The Honey Bears map, taken from the "select elements" interface.

Two interfaces were developed for this task. The two interfaces were equivalent in terms of the task structure and goals, but one was designed to be more computationally costly in cognitive terms than the other, implementing O'Hara and Payne's notion of cognitive cost and action. Specifically, the "select elements", indirect manipulation interface offers a basic map with a key showing transport routes with no visual representation of where bears, transport and honey are located. Information must be sought and moves must be issued from a separate radio-button screen (Figure 2) away from the central map. This requires the learner to explicitly seek information about the location of items, and then to maintain a mental representation of their location throughout the task. This radio button screen not only required the user to move from the map, but also broke the possible task moves into means ends analysis format, forcing the user to think explicitly about the task requirements, task goals and task moves. By contrast, the second interface, the 'drag and place', direct manipulation interface, shows where both people and transport are currently located and allows moves to be made on a focused subsection of the map by dragging and placing items (shown in Figures 3 and 4). It therefore requires less information search, and can visually represent the effects of the user's previous action.

Hence, the 'select elements' interface was identified as being more costly to the user since actions are a more lengthy diversion, require explicit search for information and a non-visually based solution structure to be devised, whilst the second, 'drag and place' interface was identified as being less costly to the user as minimal information search is required and no mental transformation of the interface elements was needed. Notably, the 'drag and place' interface also keeps error costs low by directly presenting possible moves on a subsection of the map. Only a small number of constraints remain 'implicit' in the interface. (e.g. that the pony bear is needed to drive the pony). In contrast, 'select-elements' allows radio-button selection of impossible moves (e.g. travelling in one journey from 


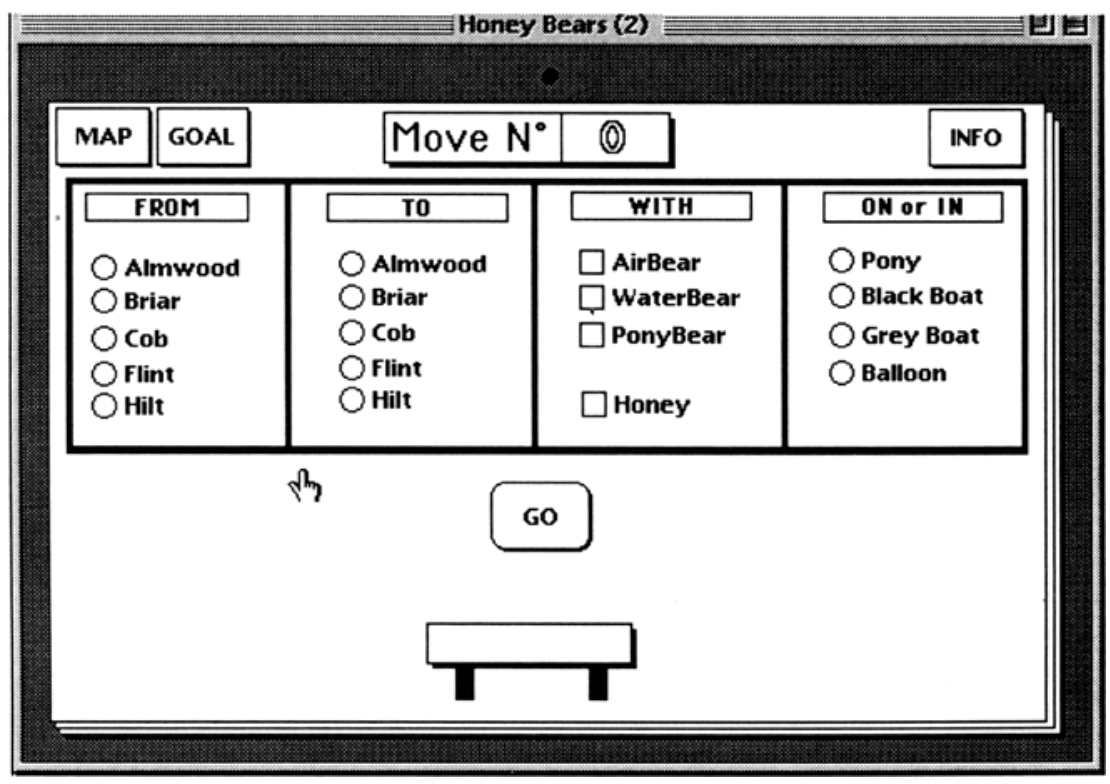

Figure 2: The "select elements" radio button interface for making moves

Flint to Almwood) and responds with an error-message only once the full set of radio-button selections is made.

An isomorphic second task was developed. Here, the bears were replaced by a king's loyal subjects, the honey monsters were replaced by pirates and the goal was to retrieve a crown. The transfer task was devised as a test of what was learnt on the Honey bears task and therefore all subjects needed to share a common interaction method; all subjects used a 'select elements' interface. For both tasks, there was one crucial piece of information which enabled the development of the correct task solution: that Honey Monsters eat honey and that pirates steal jewels, and that they should therefore be avoided by taking the balloon across the water.

\subsection{Method}

\section{Design}

A mixed design was employed. The independent variable was the type of interface experienced by subjects in their initial task, with two levels: 1) "high- cost", select-elements version of Honey bears used for the initial task and (2) "low cost", drag-and-place version of Honey bears used for the initial task. Both groups experienced the same transfer task, implemented with a 'select-elements', indirect manipulation interface. Dependent measures taken were number of information searches in total, number of information searches which were made before the first move (where information search was defined as moving to another screen to find out information about objects and locations), number of moves made before discovery of the main task constraint (that the Honey Monsters eat honey and must be avoided), percentage of move attempts which were successful (i.e. no error messages received on execution) and level reached by subjects during the trial (Levels 1 6 ). The percentage of successful moves made was a score constructed by taking $n$ (moves made without error message on execution) divided by $\boldsymbol{n}$ (total number of moves attempted, including attempted executions which failed and did result in an error message). Information searches before the first move were used as an indication that subjects were seeking to discover in advance which routes are viable (i.e. planning), rather than moving 


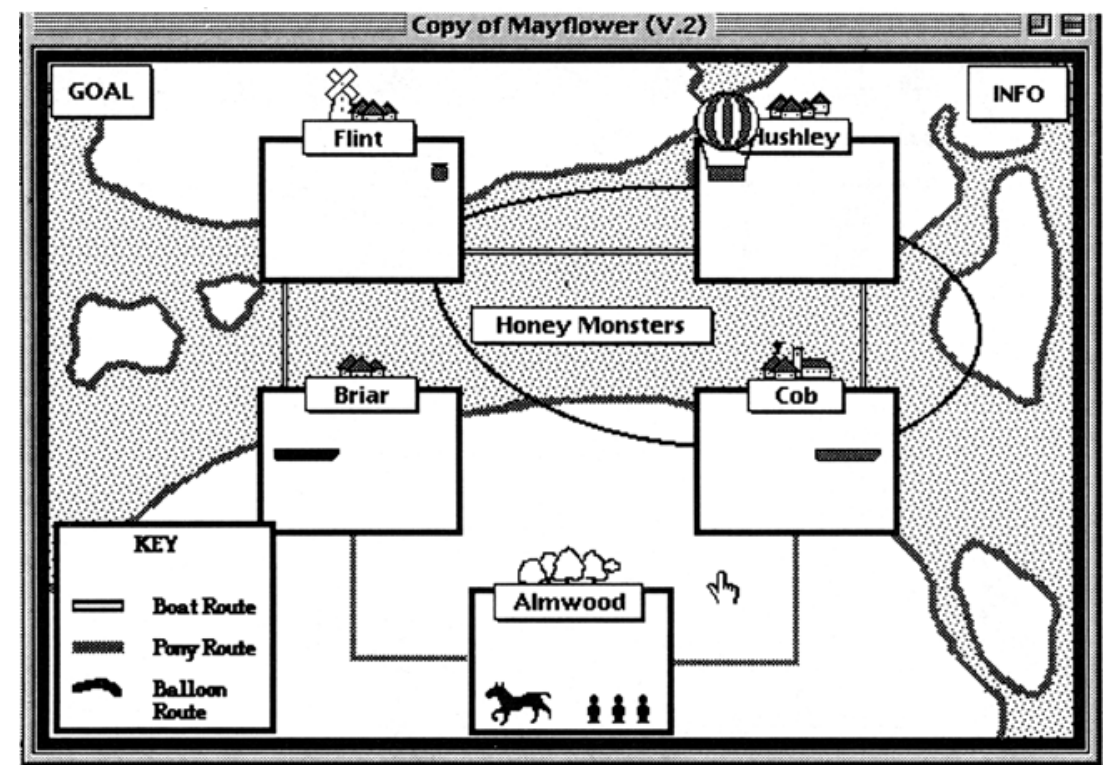

Figure 3: The Honey Bears interface, taken from the "drag and paste" interface

characters around the map by trial-and-error. Level was defined by how far they got towards delivering the honey; thus 6 was complete task success and 1 was moving the pony, the first move in an ideal task solution.

\section{Materials}

Written instructions were given to all participants, which detailed the task goals and possible actions. The tasks themselves were implemented in Hypercard on an Apple Macintosh LC II with single button mouse. Post task questionnaires asked participants to reflect on the interfaces in terms of cost.

\section{Study participants}

22 undergraduates from the University of Nottingham, aged between 18 and 23 took part in the study. Computer experience was mixed, but all had some experience of mouse-based computer interfaces. Participants were divided into two groups, with 11 participants per group.

\section{Procedure}

Study participants were randomly allocated to one of the conditions. Participants in Condition 1 trained on the "low-cost", drag-and-place version of the task before transferring to the Pirates task. Participants in Condition 2 trained on the "high-cost", select-elements version of Honey Bears before doing the Pirates task. The experimenter set up the starting screen; clicking on a START button began the session. Each participant was instructed to solve the problem as quickly as possible. Data was stored automatically to a separate Hypercard stack, as the subject completed each task. A questionnaire and debriefing followed the experimental session.

\subsection{Results}

\section{Information searches}

The 'select elements' group made a mean of 20.36 $(s d=20.63)$ information searches on the Honey Bears 


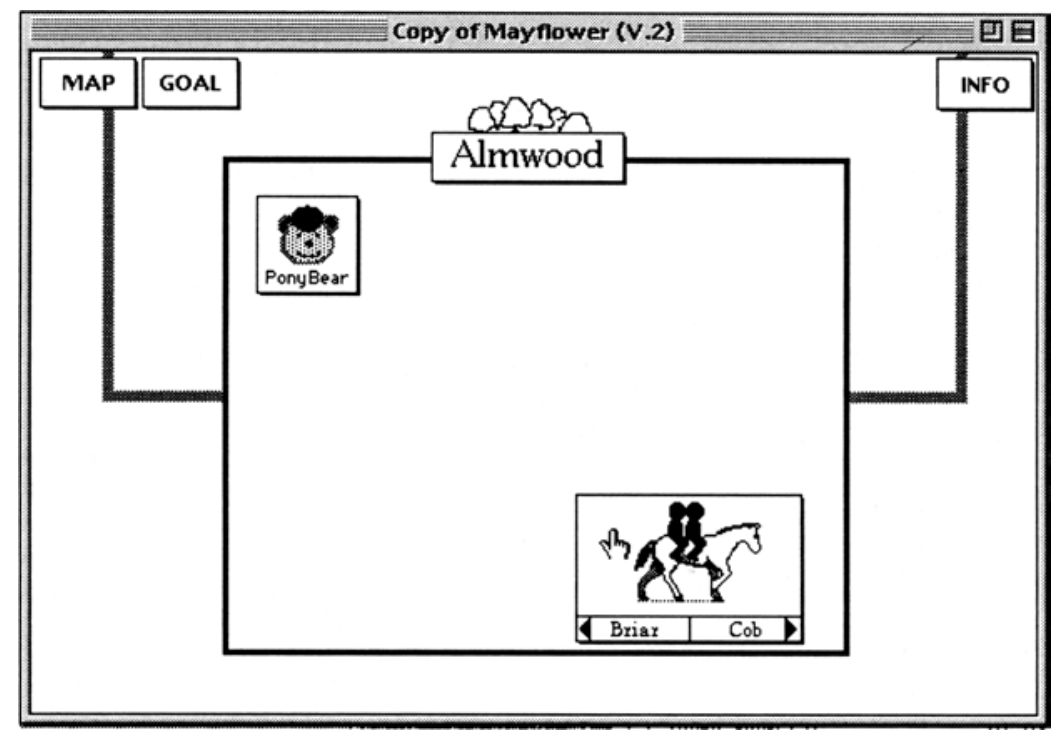

Figure 4: The Honey Bears interface: close up of the map from the 'drag-andplace' interface.

task compared to $11.73(\mathrm{sd}=10.96)$ searches on the transfer (Pirates) task. The 'drag-and-place' group made $21.27(\mathrm{sd}=12.6)$ searches at training and $27.55(\mathrm{sd}=$ 27.55) searches at transfer. An analysis of variance revealed no significant main effects but a significant interaction between group and trial $\left(\mathrm{F}_{(1,20)}=7.93\right.$, $\mathrm{p}<0.01)$.

The two experimental groups were compared for the total number of information searches made before making the first move. If information gathering can be equated with planning activities, this should enable us to distinguish how users respond to the increased cost of making a move given the alternative representations. At training, subjects using the 'select-elements', indirect manipulation interface made an average of 12.27 (sd = 15.5) information searches before making the first move, whilst subjects in the 'drag-and-place', direct manipulation group made an average of $1.41(\mathrm{sd}=4.5)$ moves before making the first move. At transfer, the 'select-elements' group made $8.46(s d=7.5)$ while the 'drag-and-place' group made $16.9(\mathrm{sd}=7.3)$ information searches before the first move. An analysis of variance revealed a significant main effect of trial $\left(F_{(1,20)}=6.62\right.$ $\mathrm{p}<.02)$ and a significant interaction between group and trial $\left(F_{(1,20)}=18.38 \mathrm{p}<0.004\right)$. These results indicate that the interface had a strong effect on the way in which subjects solved the task. The increased number of information searches before the first move made by the 'drag-and-place' group suggests that they were required to find information to solve the transfer (Pirates) task, information which the 'select elements' group had already discovered - that breaking down the goal into subgoals with constraints was an effective solution strategy.

\section{Number of moves before and after discovering the constraint}

The number of moves made before discovering the crucial information (that Honey Monsters eat honey) 
were assessed. These were compared to the number of moves made after discovering the constraint; means are presented in Table 1 . At training, an unpaired t-test revealed a significant difference between the groups on moves taken before discovering the crucial information $(t=2.83, \mathrm{p}<.01)$. This supports the assertion that the interface directed the subjects to pre-plan and search for information to a far greater extent in the 'selectelements' group than in the 'drag-and-place' group. Having found out that honey monsters eat honey and therefore a balloon must be taken across the water (requiring the presence of Airbear), the two groups were not distinguishable in their performance: there was no significant difference between the groups on number of moves made after the information has been discovered. There were no significant differences between the groups on the transfer (Pirates) task, either on moves before or moves after discovery that Pirates steal jewels. Of course, the relevance of this information had already been established through practice on the isomorphic Honey Bears task, so this is not surprising.

\begin{tabular}{|r|l|l|l|}
\hline Group & Moves & Training & Transfer \\
\hline $\begin{array}{r}\text { Select } \\
\text { elements }\end{array}$ & before & $\begin{array}{l}1.30 \\
\mathrm{sd}=1.83\end{array}$ & $\begin{array}{l}0.40 \\
\mathrm{sd}=.96\end{array}$ \\
\hline & after & $\begin{array}{l}6.3 \\
\mathrm{sd}=4.67\end{array}$ & $\begin{array}{l}8.3 \\
\mathrm{sd}=5.1\end{array}$ \\
\hline Drag & before & $\begin{array}{l}7.18 \\
\mathrm{sd}=6.3\end{array}$ & $\begin{array}{l}1.5 \\
\mathrm{sd}=2.14\end{array}$ \\
\hline & & $\begin{array}{l}6.55 \\
\mathrm{sd}=4.3\end{array}$ & $\begin{array}{l}9.0 \\
\mathrm{sd}=4.6\end{array}$ \\
\hline
\end{tabular}

Table 1. Moves before and after discovery of the main constraint

\section{Percentage of successful moves}

The percentage of successful moves reflects how many actions were taken without receiving an error message. The means for the 'select-elements' group were $46.3 \%$ (sd = 35.96) at training and $62.5 \%$ (sd = 38.5) at transfer. The means for the 'drag-and-place' group for the $73.1 \%(s d=28.2)$ at training and $86.8 \%$ $(s d=13.17)$ at transfer. There was a significant main effect of trial $\left(F_{(1,20)}=9.63, p<0.01\right)$ and a significant main effect of group $(F((1,20)=4.46 p<0.05)$. The main effect of trial shows that both groups made moves more effectively at transfer. The main effect of group reflects the extra error-constraints experienced in the 'drag-andplace' interface. Some subjects reported using the radiobutton screen to find out information: each time they triggered an error message, they gained new information about the problem. The high rate of error by the "selectelements' group during training seems to reflect use of this strategy.

\section{Level of problem solution achieved}

For the training task, the mean level achieved by the 'select-elements', indirect manipulation interface group was $3.5(\mathrm{sd}=2.2$ ) and 3.2 ( $\mathrm{sd}=2.0$ ) for the "drag-andplace' interface group. On the transfer task, participants in the 'select-elements' interface group achieved a mean score of $4.6(\mathrm{sd}=2.3)$, whilst the participants in the 'drag and place' interface group achieved a mean score of $5.4(\mathrm{sd}=1.3)$. An analysis of variance revealed a main effect of trial (Honey bears versus Pirates) $\left(F_{(1,20)}=\right.$ $7.70, \mathrm{p}<0.01)$. There was no main effect of group and no significant interaction between group and trial.

\subsection{Discussion}

It appears from these results that the interfaces did indeed result in different information search and task solution strategies for the two groups. The group presented with the 'drag and place' interface were much more inclined to use trial-and-error and took the opportunity to try things out in the interface rather than search for information or explicitly break the goal into subgoals with constraints to be satisfied. This was reflected in the greater number of moves taken before discovery of the main task constraint. Indeed, when presented with a 'select elements' interface at the transfer phase, subjects who had moved from the 'drag-and-place' interface expressed that the 'select-elements' interface was harder to use, but provided better task support by forcing thought about goals and moves. Further, the percentage of moves made without error support the notion that the 'drag and place' interface offers more information to users. In terms of learning effects, 
however, our measure of task success (level) did not provide enough support to suggest that the different manipulation styles had long lasting effects for this task. We believe this may have been due to the relative simplicity of the task, however.

\section{CONCLUSIONS}

This study has shown how laboratory based results in cognitive psychology can contribute to our understanding of how users interact with interfaces and can contribute to the development of broad brush guidelines for the development of learning interfaces. It also illustrates, however, that results from psychological studies cannot be taken off-the-shelf but must be put into clear context. Specifically, we believe that this study raises the need for a clearer definition of "cognitive cost" than that offered by O'Hara and Payne and by Svendsen. O'Hara and Payne's definitions of cost were so broad as to range from physical, to strategic, to mental costs. There are a number of different types of cost, with potentially very different effects on learner strategies. The effects of cost in the two Honey Bears interfaces ranged from provoking information search, to memory strategies, planning ahead to avoid wasted effort, and finally, mere diversion away from the domain in favour of the interface itself.

The issues of cost and of information presentation, and information available at the time of making a move will be developed in subsequent studies. The educational value of costliness in an interface seems to lie in its directive properties; such that the manipulation style encourages attention to be given to certain domain relationships rather than others. Cost will be beneficial to learning when it induces cognitive mechanisms which are integral in the learning process. Conversely, cost will be damaging to learning when it induces cognitive mechanisms which are superfluous to the learning process and the worst possible scenario is when superfluous cognitive processes are highly demanding on the learner's limited attentional resources.

\section{ACKNOWLEDGEMENTS}

This work was completed as part of a doctoral thesis, supported by EPSRC grant 94315041 . The second author is supported by a EPSRC grant GR/J97786.

\section{REFERENCES}

Churchill, E. F., \& Ainsworth, S. (1995). "Making Claims about Teaching Systems." in K Norby, P.H. Helmersen, D.J. Gilmore, S.A. Arnesen (Eds) HumanComputer Interaction. Interact '95. Chapman \& Hall

Corbett, Anderson, J. R., \& Fincham (1990). "Menu Selection versus Typing: Effects on Learning in an Intelligent Programming Tutor." in C. Frasson \& G. Gauthier (Eds) Intelligent Tutoring Systems: At the Crossroads of Artificial Intelligence \& Education.

Gilmore, D. (1996) The Relevance of HCI Guidelines for Educational Interfaces. Machine Mediated learning, 5 (2) 119-133.

Jackson, S., Stratford, S.J., Krajcik, J and Soloway, E. (1996) A Learner-centred Tool for Students Buidling Models. In Communications of the ACM,: Special issue on Learner Centred Design 39, 4, 24-27.

Laurillard, D. (1993) Rethinking University teaching: A Framework for the effective use of educational technology. London: Routledge.

Littleton, K., Light, P., Joiner, R., Messer, D and Barnes, P. (1994) Gender and software interaction in children's computer-based problem solving. Internal report: ESRC Centre for Research in Development, Instruction and Training, Department of Psychology, University of Nottingham, Nottingham, UK.

Malone, T. (1981). "Toward a theory of intrinsically motivating instruction." Cognitive Science 4: 333-369.

Moore, A. (1993) Siuli's Maths Lesson: Autonomy or Control. In J. Benyon and H. Mackay (eds) Computers into Classroom. More Questions Than Answers. The Falmer Press, Sussex, UK.

Norman, D. and Spohrer, J. (1996) Introduction. Communications of the ACM: Special issue on Learner Centred Design, 39, 4, 24-27.

O'Hara, K. and Payne, S (1994). "Cost of operations affects planfulness of action." Unpublished internal report, School of Psychology, Cardiff College, University of Wales, Wales, UK.

Svendsen, G. B. (1991). "The influence of interface style on problem solving." International Journal of Man-Machine Studies 35: 379-397. 